\title{
Early Vitamin K Deficiency Bleeding in a Neonate Associated with Maternal Acute Fatty Liver of Pregnancy
}

\author{
Shreyas Arya, MBBS ${ }^{1}$ Carol J. Richardson, MD² Sunil Jain, MD² Leonard E. Swischuck, MD ${ }^{3}$
}

${ }^{1}$ Department of Pediatrics, University of Texas Medical Branch Galveston, Galveston, Texas

2 Department of Pediatrics, Division of Neonatology, University of Texas Medical Branch Galveston, Galveston, Texas

${ }^{3}$ Department of Radiology, University of Texas Medical Branch Galveston, Galveston, Texas

\author{
Address for correspondence Shreyas Arya, MBBS, Department of \\ Pediatrics, University of Texas Medical Branch Galveston, Resident, \\ PGY-2, 301 University Blvd., Galveston, TX 77555-0354 \\ (e-mail: sharya@utmb.edu).
}

Am J Perinatol Rep 2015;5:e193-e195.

\author{
Abstract \\ Keywords \\ - acute fatty liver of \\ pregnancy \\ - vitamin K-deficiency \\ bleeding \\ - hepatic dysfunction \\ - intracranial bleeding
}

Introduction Acute fatty liver of pregnancy (AFLP) is a rare but potentially fatal condition occurring in the third trimester or early postpartum period. It is characterized by microvesicular fatty infiltration of the liver. Clinically, the three most prominent derangements in women with AFLP are hepatic dysfunction, renal insufficiency, and impaired coagulation. AFLP is associated with an increased incidence of morbidity and mortality in neonates, though the exact cause for this remains unclear. Deficiency of vitamin $\mathrm{K}$ in patients with liver disease has been widely reported.

Case Description We present a unique case of severe intracranial bleeding because of the early vitamin $\mathrm{K}$ deficiency in a neonate whose mother had AFLP along with accompanying renal insufficiency.

Conclusion We suggest that monitoring infants born to mothers with AFLP, for vitamin $\mathrm{K}$ deficiency bleeding will help reduce morbidity and mortality in these infants.
An African American male infant was born via emergency cesarean delivery for prolapsed cord at 33 weeks' gestation to a 22-year-old primigravida female with negative serologies (Hepatitis B, Syphilis, and HIV) and unknown Group B Streptococcus status with no treatment. She presented with a 3week history of nausea and vomiting. She was diagnosed with acute fatty liver of pregnancy (AFLP) elevated aspartate aminotransferase (AST) 393 units/L (range, 13-40 units/L), alanine aminotransferase (ALT) 429 units/L (range, 9-51 units/L), unconjugated bilirubin $2.5 \mathrm{mg} / \mathrm{dL}$ (range, 0.1-1.1 $\mathrm{mg} / \mathrm{dL}$ ), prothrombin time (PT) 14.4 seconds (range, 1214.7 seconds), activated partial thromboplastin time (aPTT) 25 seconds (range, 23-38 seconds) and fibrinogen of $721 \mathrm{mg} /$ $\mathrm{dL}$ (range, 214-470 $\mathrm{mg} / \mathrm{dL}$ ) along with accompanying renal insufficiency creatinine $4.2 \mathrm{mg} / \mathrm{dL}$ (range, $0.50-1.04 \mathrm{mg} / \mathrm{dL}$ ). Following delivery, the mother's liver function, renal func- tion, and coagulation studies gradually improved and she was discharged 4 days following her delivery without any longterm adverse outcomes.

The infant had heart rate decelerations before birth and had respiratory depression, weak reflex response, low tone, and heart rate $<60$ beats/minute at birth. Initiation of bag mask ventilation resulted in improved heart rate $(>100$ beats/minute) and color. The baby continued to have poor respiratory effort at 2 to 3 minutes and required intubation and mechanical ventilation. The Apgar scores were 1, 6, and 7 at 1,5 , and 10 minutes, respectively. The baby weighed $1,630 \mathrm{~g}$ at birth which was appropriate for his gestational age.

In the neonatal intensive care unit, the baby showed continued bleeding from the venipuncture site. Coagulation studies showed elevated PT 98.5 seconds (range, 1214.7 seconds), aPTT 104 seconds (range, 23-38 seconds) and received

December 12, 2014 accepted after revision May 28, 2015 published online September 7, 2015
Dol http://dx.doi.org/ 10.1055/s-0035-1557107. ISSN 2157-7005.
Copyright (c) 2015 by Thieme Medical Publishers, Inc., 333 Seventh Avenue, New York, NY 10001, USA. Tel: +1(212) 584-4662.
License terms

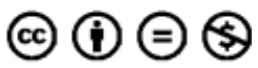


slightly low fibrinogen $184 \mathrm{mg} / \mathrm{dL}$ (range, 214-470 mg/dL). A complete blood count showed significantly low hemoglobin $9.9 \mathrm{~g} / \mathrm{dL}$ (range, 9.5-13.5 g/dL) and hematocrit 29.6\% (range, $29-41 \%)$ and platelets were $174 \times 10^{3} / \mathrm{mm}^{3}\left(150-328 \times 10^{3} /\right.$ $\mathrm{mm}^{3}$ ). Liver function tests at 7 hours of life showed a slightly elevated AST at 65 units/L but was otherwise normal-ALT 24 units/L, alkaline phosphatase 160 units/L, albumin $2.7 \mathrm{~g} / \mathrm{dL}$ (range, 3.5-5 g/dL), total protein $4.4 \mathrm{~g} / \mathrm{dL}$ (range, 4.6-7.3 g/dL), total bilirubin $3.1 \mathrm{mg} / \mathrm{dL}$, and unconjugated bilirubin $2.7 \mathrm{mg} /$ dL. A blood culture was also obtained before starting empiric antibiotics, which was negative. The baby received a routine $1 \mathrm{mg}$ dose of vitamin $\mathrm{K}$ intramuscularly following which coagulation panel was repeated which showed PT of $58 \mathrm{sec}-$ onds and aPTT of 104 seconds. The baby also received a $20 \mathrm{~mL} /$ $\mathrm{kg}$ packed red blood cell transfusion following which the repeat hemoglobin and hematocrit improved to $14.2 \mathrm{~g} / \mathrm{dL}$ and $40.3 \%$. The baby had seizure activity which was confirmed on electroencephalography and treated with phenobarbital and fosphenytoin. A computerized tomography scan of the head in the first 24 hours following birth showed a grade 4 intraventricular hemorrhage with subdural bleeding (-Fig. 1). Baby had normal factor 8 levels of 2.12 units $/ \mathrm{mL}$ (range, $0.47-1.70$ units $/ \mathrm{mL}$ ) and slightly low factor 9 of 0.06 units/mL (range, $0.76-1.58$ units $/ \mathrm{mL}$ ), and 10 levels of 0.17 units/mL (range, 0.76-1.49 units/mL) not consistent with hemophilia. The baby was given a $5 \mathrm{mg} / \mathrm{kg}$ dose of vitamin $\mathrm{K}$ intravenously which resulted in the prompt normalization of the coagulation studies PT 21.3 seconds/aPTT 46 seconds and fibrinogen $302 \mathrm{~mL} / \mathrm{dL}$ and the bleeding from puncture sites resolved which is consistent with the diagnosis of early vitamin K-deficiency bleeding (VKDB).

A repeat computerized tomography scan at 40 weeks of age showed markedly enlarged lateral ventricles secondary to loss of brain parenchyma and severe encephaomalacia (-Fig. 2). At the follow-up visits, the child was found to have global developmental delays with feeding intolerance which

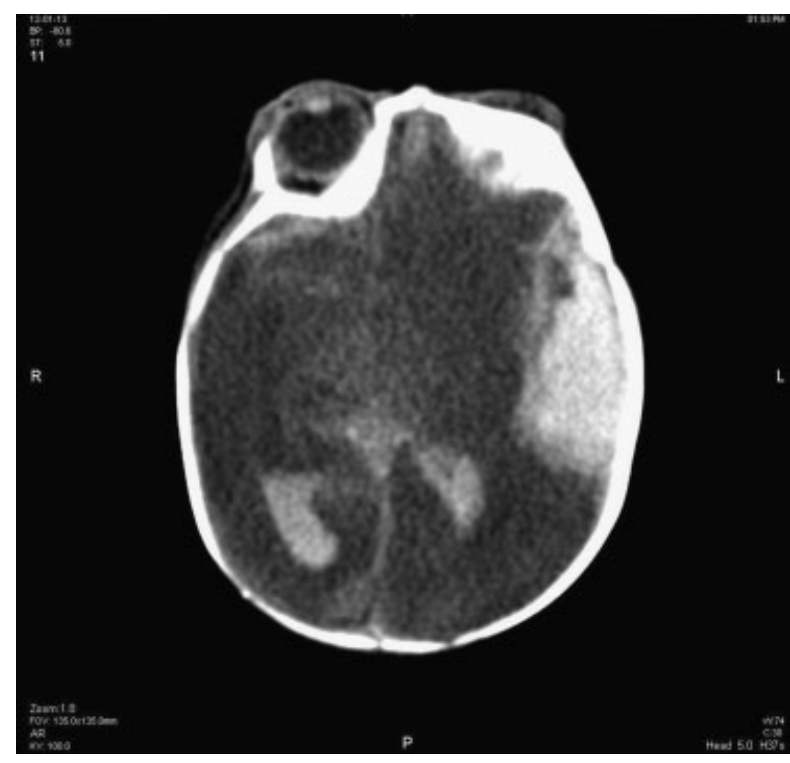

Fig. 1 Computerized tomography scan of at 24 hours of life showing diffuse cerebral edema, subdural collection on the left, midline shift, and blood in both occipital horns.

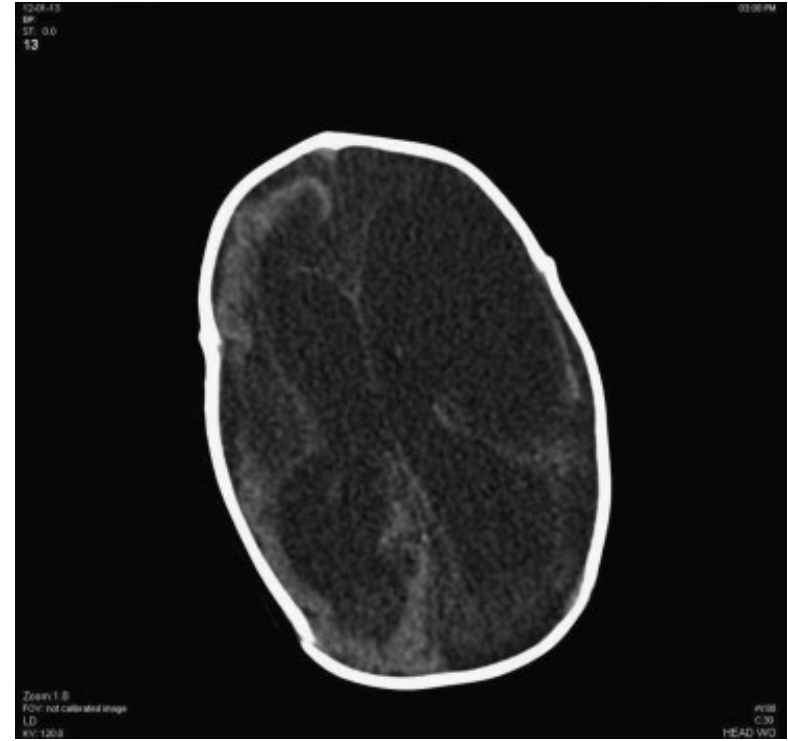

Fig. 2 Computerized tomography scan of the head at 40 weeks showing markedly enlarged lateral ventricles secondary to loss of brain parenchyma and severe encephalomalacia.

required a Nissen's fundoplication and G-tube placement. The infant continues to be followed by our neonatal follow-up clinic and has not had any other concerns with regards to bleeding till date.

\section{Discussion}

Even though there was an extensive body of work on the relationship between bleeding in newborns and vitamin $\mathrm{K}$ earlier, the disease was seen infrequently. With the increased push toward exclusive breast feeding, it started appearing more and more. This was evidenced by the 1983 Lancet article "Hemorrhagic Disease of the Newborn returns."1

Over the past few years, the term "Hemorrhagic disease of the newborn" has been replaced by "Vitamin K-deficiency bleeding" because there are other etiologies of bleeding in the newborn and VKDB can often present after 4 weeks.

Today, it is the standard of care around the world to provide vitamin $\mathrm{K}$ supplementation to the newborn at birth. The American Academy of Pediatrics (AAP) endorses universal supplementation with a single intramuscular dose (range, $0.5-1 \mathrm{mg}$ ) of vitamin K within 1 hour of birth. ${ }^{2}$

Like vaccinations, parenteral vitamin $\mathrm{K}$ was also met with opposition from parents early on, especially because of an unproven assumption that older phenol containing preparations of vitamin $\mathrm{K}$ were associated with childhood cancer (leukemia) later in life. This even led to some countries adopting oral administration of vitamin $\mathrm{K}$ to newborns. But increased incidences of late type bleeding has led to oral vitamin $\mathrm{K}$ falling out of favor. In fact, no oral preparations of vitamin $\mathrm{K}$ are licensed for use in the United States. Also, the newer formulation of vitamin $\mathrm{K}$ is solubilized in natural components (glycocholic acid and phosphatidylcholine) which has improved the safety profile and has a much lower risk of serious anaphylactoid reactions. ${ }^{3}$ 
Vitamin $\mathrm{K}$ is an essential lipid soluble vitamin which is involved in the gamma carboxylation of glutamate residues which are found in coagulation proteins (factors II, VII, IX, and $\mathrm{X}$ and protein $\mathrm{C}, \mathrm{S}$, and Z). VKDB is defined as bleeding because of the deficiency of vitamin $\mathrm{K}$-dependent clotting factors. It presents initially as ecchymosis, petechia, easy bruisability, or spontaneous bleeding from mucosal sites. However, it is most often discovered because of the presence of continued bleeding from venipuncture and circumcision sites or from the cut umbilical cord. A prolonged PT with normal fibrinogen levels and platelet count is diagnostic of VKDB and a rapid correction of PT with cessation of bleeding after vitamin K administration is confirmative. Overall, 30 to $60 \%$ cases of VKDB are associated with intracranial hemorrhages ${ }^{4}$ which is why early identification and treatment is essential. Elalfy et al in 2014 found that all 21 infants with intracranial hemorrhage in the neonatal period in their study were found to have significant vitamin $\mathrm{K}$ deficiency. They also found that administering vitamin $\mathrm{K}$ to these patients corrected their PT and PTT values within 48 hours and highly significant negative correlation was found between serum vitamin K levels and PT and PTT values. Also, the most prevalent site of bleeding in these infants was subdural hemorrhage. ${ }^{5}$

VKDB is classified based on the age of onset as: early $(<24$ hours after birth); classic (1-7 days after birth), and late ( 1 week to 6 months after birth). The etiology of VKDB is either idiopathic or secondary.

Most cases of early VKDB are attributable to drugs taken by the mother during pregnancy. These medications include anticonvulsants (carbamazepine, phenytoin, and barbiturates), antibiotics (cephalosporin), antitubercular drugs (rifampin and isoniazid) and vitamin K antagonists (coumarin). ${ }^{6}$ However, the mother, in the case we are reporting, was not on any of these medications.

AFLP is a rare but serious condition. It is characterized by microvesicular fatty infiltration of the liver. The condition is associated with an inherited deficiency of a mitochondrial enzyme-long chain 3-hydroxyacetyl coenzyme-A dehydrogenase (LCHAD) but other enzymes have been implicated as well. The infant in our case had normal results for LCHAD on newborn screening. The diagnosis of AFLP can be made by using the Swansea Criteria. Clinically, the three most prominent derangements in women with AFLP are hepatic dysfunction, renal insufficiency, and impaired synthesis of procoagulants. $^{7}$ AFLP is associated with an increased incidence of morbidity and mortality in neonates.

It also is important to differentiate AFLP from the more commonly seen HELLP syndrome. Liver failure and the accompanying complications such as decreased procoagulant synthesis are rarely found in HELLP syndrome and are essential for the diagnosis of AFLP. Elevated serum creatinine values are also more commonly associated with AFLP. Pathogenesis of acute renal failure in AFLP is not well understood. ${ }^{8}$
Deficiency of vitamin $\mathrm{K}$ in patients with liver disease has been widely reported. In fact, vitamin $\mathrm{K}$ repletion is a commonly used procoagulant strategy in these patients. Importantly, prothrombin time is a late indicator of vitamin $\mathrm{K}$ deficiency and can be normal even when prothrombin concentrations fall to half normal values. ${ }^{9}$ This explains the normal maternal PT even in the presence subclinical vitamin $K$ deficiency. While the water soluble vitamins are actively transported across the placenta, the fat soluble vitamins (A, D, $\mathrm{E}$, and $\mathrm{K}$ ) are transferred to the fetus by facilitated diffusion, at a much slower rate. ${ }^{10}$ So, maternal conditions causing sub clinical vitamin deficiencies will result in overt symptoms in the neonate.

In the past, the neonatal mortality rate had been estimated to be as high as $85 \%$; however, with prompt recognition and treatment, the mortality rate has dramatically decreased to approximately $23 \%{ }^{11}$ We suggest to monitor infants born to mothers with AFLP for possible VKDB as prompt management will help avoid devastating intracranial bleeding and reduce long-term morbidities.

\section{Conflict of Interest}

The authors declare no conflict of interest.

\section{References}

1 McNinch AW, Orme RL, Tripp JH. Haemorrhagic disease of the newborn returns. Lancet 1983;1(8333):1089-1090

2 American Academy of Pediatrics Committee on Fetus and Newborn. Controversies concerning vitamin $\mathrm{K}$ and the newborn. Pediatrics 2003;112(1 Pt 1):191-192

3 Clarke P, Shearer MJ. Vitamin K deficiency bleeding: the readiness is all. Arch Dis Child 2007;92(9):741-743

4 Sutor AH. Vitamin K deficiency bleeding in infants and children. Semin Thromb Hemost 1995;21(3):317-329

5 Elalfy MS, Elagouza IA, Ibrahim FA, AbdElmessieh SK, Gadallah M. Intracranial haemorrhage is linked to late onset vitamin $\mathrm{K}$ deficiency in infants aged 2-24 weeks. Acta Paediatr 2014;103(6): e273-e276

6 Cornelissen M, Steegers-Theunissen R, Kollée L, et al. Increased incidence of neonatal vitamin $\mathrm{K}$ deficiency resulting from maternal anticonvulsant therapy. Am J Obstet Gynecol 1993;168(3 Pt 1): 923-928

7 Nelson DB, Yost NP, Cunningham FG. Acute fatty liver of pregnancy: clinical outcomes and expected duration of recovery. Am J Obstet Gynecol 2013;209(5):456.e1-456.e7

8 Koroshi A, Babameto A. Acute renal failure during acute fatty liver of pregnancy. Nephrol Dial Transplant 2002;17(6):1110-1112

9 Sokoll LJ, Sadowski JA. Comparison of biochemical indexes for assessing vitamin $\mathrm{K}$ nutritional status in a healthy adult population. Am J Clin Nutr 1996;63(4):566-573

10 Malone JI. Vitamin passage across the placenta. Clin Perinatol 1975;2(2):295-307

11 Fesenmeier MF, Coppage KH, Lambers DS, Barton JR, Sibai BM. Acute fatty liver of pregnancy in 3 tertiary care centers. Am J Obstet Gynecol 2005;192(5):1416-1419 